\title{
CONE-ANGLE PARAMETRIZATION OF THE ARRAY MANIFOLD in DF SYSTEMS
}

\author{
by \\ H.R. Karimi and A. Manikas \\ Department of Electrical \& Electronic Engineering \\ Imperial College of Science, Technology and Medicine \\ London
}

\section{Submitted to: The Franklin Institute, Engineering and Applied Mathematics 15th August 1996 \\ (Accepted for Publication)}

\begin{abstract}
The manifold of a planar array in a direction finding system may be considered as two families of azimuth $\theta$ and elevation $\phi$ curves, where the $\phi$ parameter curves are hyperhelical as well as geodesic while the $\theta$-parameter curves are neither. Since the $\theta$-curves are not hyperhelical, their curvatures depend on $\theta$ and so analytical evaluation of curvatures of order greater than two can become exceedingly laborious and impractical. The advantages of having hyperhelical parameter curves are numerous. For one thing, all the curvatures of a hyperhelix may be evaluated recursively (since they do not vary from point to point) as a function of lower-order curvatures. This has been demonstrated in [1] for the case of the singleparameter manifold of a linear array. Furthermore the convenient nature of a hyperhelix's geometry has proven invaluable in array design [2], in investigating the detection and resolution thresholds [3] and in identifying ambiguities inherent in array configurations [4]. In view of the above facts, it seems logical that an alternative parametrization of the manifold surface, which results in two sets of hyperhelical parameter curves, can provide a great deal of additional insight into the nature of planar array behaviour and design. In this investigation, such a parametrization is identified and its significance is demonstrated by a number of examples/applications. Furthermore properties, such as Gaussian and geodesic curvatures, are defined and their implications with regards to isometric mappings are discussed.
\end{abstract}




\section{Nomenclature}

$\begin{array}{llll}A, a & \text { Scalar } & \mathcal{L}[\mathbb{A}] & \text { Space spanned by columns of } \\ \mathbb{A} & \text { Vector } & \exp \{\underline{a} \text { or } \mathbb{A}\} & \text { Element by element exponential } \\ \underline{A}, \underline{\mathrm{a}} & \text { Matrix } & \operatorname{sum}(\underline{\mathrm{a}}) & \text { Sum of vector elements } \\ \underline{\mathbb{A}}, \underline{\mathbf{A}} & \text { Absolute value of scalar } & \underline{\mathrm{a}}^{k} & \text { Element by element } k^{\text {th }} \text { power } \\ |a| & p & \text { General } \\ |\underline{\mathrm{a}}| & \text { Euclidian norm of vector } & \dot{a} & \text { Derivative } \partial a / \partial p \\ \text { parameter } & & a^{\prime} & \text { Derivative } \partial a / \partial s \\ \operatorname{Re}(\bullet) & \text { Real part } & & \end{array}$

\section{Introduction}

Consider the manifold vector, representing the response of a planar array of $N$ isotropic sensors receiving a unity power signal from a far-field emitter,

$$
\underline{\mathrm{a}}=\exp \left\{-j \underline{\underline{\mathbf{r}}}^{\mathrm{T}} \underline{k}\right\}
$$

where $\underline{\underline{\mathbf{r}}}=\left[\underline{r}_{x}, \underline{r}_{y}, \underline{0}\right]^{\mathrm{T}} \in \mathcal{R}^{N \times 3}$ is the matrix of sensor locations in units of halfwavelengths $(\lambda / 2)$ and $\underline{k}$ is the wavenumber vector pointing towards the emitter. The locus of manifold vectors for all emitter bearings is known as the array manifold.

It is common practice in the array processing literature to specify the direction of arrival of a received signal by its azimuth $\theta$ (angle made with the $x-z$ plane) and elevation $\phi$ (angle made with the $x$-y plane), in which case the wavenumber vector is given by

$$
\underline{k}(\theta, \phi)=\pi[\cos \phi \cos \theta, \cos \phi \sin \theta, \sin \phi]^{\mathrm{T}}
$$

In spite of the intuitive appeal of using azimuth and elevation, this choice of angles is by no means unique and furthermore is not the most suitable for the study of the array manifold of planar arrays. A plane-wave may be characterized by any two independent angles which specify its orientation with respect to an arbitrary frame. For example, consider the frame $\stackrel{v}{x}-\stackrel{v}{y}$ - $z$ where $\stackrel{v}{x}$ and $y$ are the result of rotating the $x$ and $y$ axes by an angle $\Theta$. Then, defining $\alpha$ and $\beta$ as the bearings of the arriving wavefront with respect to the positive directions of the $\stackrel{v}{x}-\stackrel{v}{y}$-axes respectively, the wavenumber vector $\stackrel{v}{\underline{k}}$ may be written as :

$$
\begin{aligned}
& \stackrel{\stackrel{v}{k}}{(\alpha, \beta)}=\pi\left[\cos \alpha, \cos \beta, \sqrt{1-\cos ^{2} \alpha-\cos ^{2} \beta}\right]^{\mathrm{T}} \\
& \text { where } \begin{cases}\cos \alpha=\cos \phi \cos (\theta-\Theta) & ; 0 \leq \alpha \leq \pi \\
\cos \beta=\cos \phi \sin (\theta-\Theta) & ; 0 \leq \beta \leq \pi\end{cases}
\end{aligned}
$$

The relationship between the $\theta-\phi$ and $\alpha-\beta$ conventions is illustrated in Fig. (1a). At this stage the purpose of rotation angle $\Theta$ may be unclear, however this will become apparent in subsequent sections. As can be seen, $\alpha$ and $\beta$ take values between 0 and $\pi$ as they are defined according to their cosines. Parameters $\alpha$ and $\beta$ are also known as cone angles since the loci of wavenumber vectors of constant $\alpha$ (or $\beta$ ) form a cone about the $\tilde{x}$ (or $\tilde{y}$ ) axis as shown in Fig. (1b). 

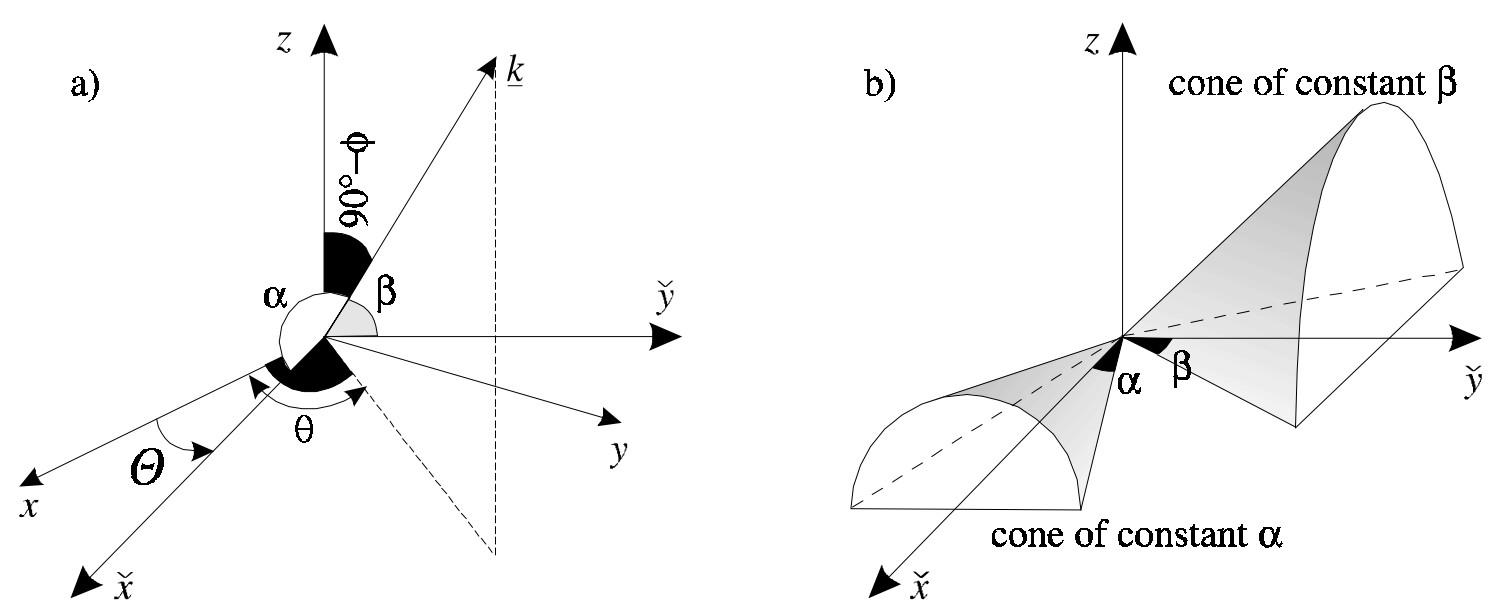

Fig.(1): $\quad$ a) The $\theta-\phi$ and $\alpha-\beta$ conventions.

b) Cones formed by wavenumber vectors of constant $\alpha$ and constant $\beta$.

Fig. (2) illustrates the one-to-one mapping $(\theta, \phi) \mapsto(\alpha, \beta)$ specified by Eq. (4). It can be observed that $\alpha$ and $\beta$ can vary independently, if and only if, $\phi \neq 0$. Furthermore it should also be pointed out that, unlike $\theta$ and $\phi$, not all combinations of $\alpha$ and $\beta$ are possible. For example:

$$
\text { if } \alpha=\alpha_{1} \text { then } \begin{cases}\frac{\pi}{2}-\alpha_{1} \leq \beta \leq \frac{\pi}{2}+\alpha_{1} & \text { for } \alpha_{1} \leq \frac{\pi}{2} \\ \frac{\pi}{2}-\left(\pi-\alpha_{1}\right) \leq \beta \leq \frac{\pi}{2}+\left(\pi-\alpha_{1}\right) & \text { for } \alpha_{1}>\frac{\pi}{2}\end{cases}
$$
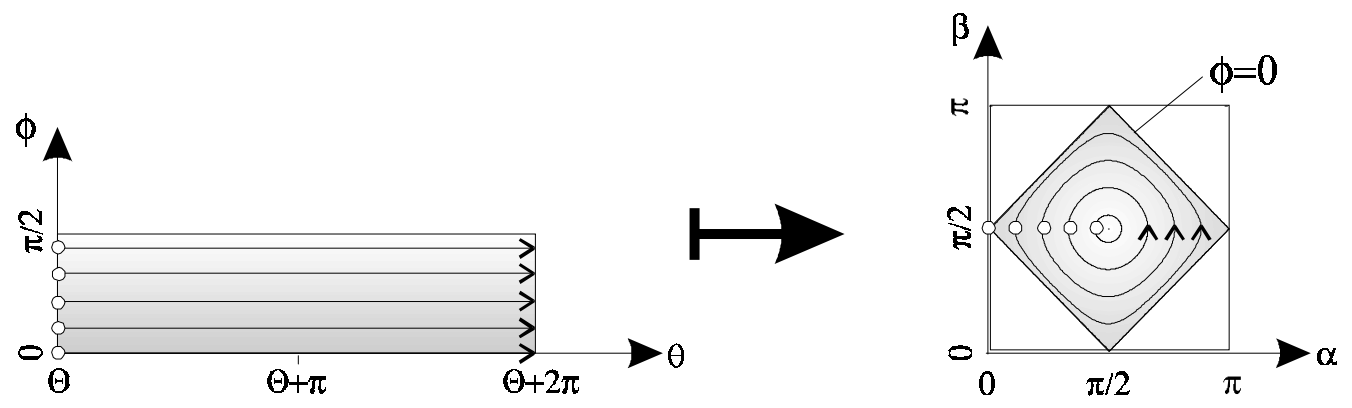

Fig.(2) : Mapping from the $(\theta, \phi)$ parameter space on to the $(\alpha, \beta)$ parameter space.

The rotation of the $x-y$ frame by $\Theta$ implies that matrix $\stackrel{\vee}{\underline{\mathbf{r}}}$ of sensor locations with respect to the $\stackrel{\vee}{x}$ and $y$ axes is given by :

$$
\stackrel{\stackrel{\vee}{\underline{r}}}{=}\left[\underline{R}(\Theta), \underline{R}\left(\Theta+\frac{\pi}{2}\right), \underline{0}\right]^{\mathrm{T}}
$$


where $\underline{R}(\Theta)=\underline{r}_{x} \cos \Theta+\underline{r}_{y} \sin \Theta$. The manifold of a planar array of isotropic sensors, parametrized according to cone angles $\alpha$ and $\beta$, may then be written as :

$$
\begin{aligned}
\underline{\mathrm{a}}(\alpha, \beta) & =\exp \{-j \underline{\underline{\mathbf{r}}} \stackrel{\mathrm{T}}{\mathrm{v}}(\alpha, \beta)\} \\
& =\exp \left\{-j \pi\left(\underline{R}(\Theta) \cos \alpha+\underline{R}\left(\Theta+\frac{\pi}{2}\right) \cos \beta\right)\right\}
\end{aligned}
$$

It should be emphasized here that $\Theta$ is a fixed scalar and not a direction of arrival parameter. Angles $\Theta$ and $\Theta+\frac{\pi}{2}$ simply define the lines of azimuth with respect to which $\alpha$ and $\beta$ are respectively measured.

It is the objective of this paper to investigate the array manifold surface using coneangle parametrization which can provide a great deal of additional insight into the behaviour of DF systems employing planar arrays. Section II contains a summary of those results of differential geometry which are of practical interest in the study of array manifolds of planar arrays and the performance of direction-finding array systems. These results are subsequently used in Sections III and IV for investigating the individual $\alpha$ - and $\beta$-parameter curves as well as the intrinsic geometry of the manifold surface. Section V examines the implications of the geometry of the $\alpha$ - and $\beta$-parameter curves in connection with the direction-finding capabilities of a planar array of sensors. The paper finally concludes in Section VI. 


\section{Differential Geometry of a Two-Parameter Manifold (Surface) in Complex $N$-Space}

Definition - A regular parametric representation of a surface is defined as a vector function

$$
\underline{\mathrm{a}}=\underline{\mathrm{a}}(p, q)
$$

of parameters $p$ and $q$ in the parameter space $\Omega$ iff $, \forall p, q \in \Omega$, the derivatives $\partial \underline{\mathrm{a}} / \partial p$ and $\partial \underline{\mathrm{a}} / \partial q$ exist, are continuous and non-zero with

$$
\operatorname{rank}\left\{\left[\frac{\partial \mathrm{a}}{\partial p}, \frac{\partial \mathrm{a}}{\partial q}\right]\right\}=2
$$

The curve $\underline{\mathrm{a}}\left(p, q_{\mathrm{o}}\right)$ which joins all those points on the manifold surface corresponding to a constant value of $q=q_{\mathrm{o}}$ is referred to as a $p$-parameter curve. Different values of $q_{\mathrm{o}}$ generate a family of $p$-parameter curves covering the whole of the manifold surface. In a similar way, a $q$-parameter curve $\underline{a}\left(p_{0}, q\right)$ joins all those points on the surface corresponding to a constant value of $p=p_{\mathrm{o}}$ and, again, different values of $p_{\mathrm{o}}$ result in a family of $q$-parameter curves.

Vectors $\underline{\mathrm{a}}_{p}=\partial \underline{\mathrm{a}} / \partial p$ and $\underline{\mathrm{a}}_{q}=\partial \underline{\mathrm{a}} / \partial q$ at a point $A$ on the manifold represent the tangent vectors to the $p$ - and $q$-parameter curves respectively passing through $A$ and also form a basis for the tangent plane to the surface at that point, i.e.

$$
\text { Tangent plane }=\mathcal{L}\{\mathbb{T}\} \quad \text { where } \quad \mathbb{T}=\left[\underline{a}_{p}, \underline{a}_{q}\right] \in \mathcal{C}^{N \times 2}
$$

The regularity condition of Eq. (9) ensures that a tangent plane exists at all points on the surface. Note that the basis created by $\mathbb{T}$ is in general not orthonormal.

In the same way that the shape of a 1-parameter manifold (curve) is uniquely defined by its curvatures [1], the shape of a 2-parameter manifold (surface) may be quantitatively expressed in terms of intrinsic properties such as its Gaussian curvature and the geodesic curvature of the curves lying on its surface. In order to evaluate these quantities, it is first necessary to introduce the building blocks of surface differential geometry as exemplified by the first fundamental coefficients and the Christoffel symbols.

\subsection{The first fundamental form and coefficients}

Let $\underline{\mathrm{a}}=\underline{\mathrm{a}}(p, q)$ be the regular parametric representation of a surface. Then the differential mapping $\mathrm{da}=\underline{\mathrm{a}}_{p} \mathrm{~d} p+\underline{\mathrm{a}}_{q} \mathrm{~d} q$ maps increments $\mathrm{d} p$ and $\mathrm{d} q$ on the $(p, q)$ parameter plane on to vector $\underline{\mathrm{a}}_{p} \mathrm{~d} p+\underline{\mathrm{a}}_{q} \mathrm{~d} q$ on the tangent plane at $\underline{\mathrm{a}}(p, q)$. The scalar I, defined as

where

$$
\mathrm{I}=\|\mathrm{d} \underline{\mathrm{a}}\|^{2}=\left\|\underline{\mathrm{a}}_{p} \mathrm{~d} p+\underline{\mathrm{a}}_{q} \mathrm{~d} q\right\|^{2}=[\mathrm{d} p, \mathrm{~d} q] \mathbb{G}\left[\begin{array}{l}
\mathrm{d} p \\
\mathrm{~d} q
\end{array}\right]
$$




$$
\mathbb{G}=\operatorname{Re}\left(\mathbb{T}^{\mathrm{H}} \mathbb{T}\right)=\left[\begin{array}{ll}
g_{p p} & g_{p q} \\
g_{q p} & g_{q q}
\end{array}\right]=\left[\begin{array}{cc}
\left\|\underline{\mathrm{a}}_{p}\right\|^{2} & \operatorname{Re}\left(\underline{\mathrm{a}}_{p}^{\mathrm{H}} \underline{\mathrm{a}}_{q}\right) \\
\operatorname{Re}\left(\underline{\mathrm{a}}_{q}^{\mathrm{H}} \underline{\mathrm{a}}_{p}\right) & \left\|\underline{\mathrm{a}}_{q}\right\|^{2}
\end{array}\right]
$$

is known as the first fundamental form and essentially represents the distance between two neighboring points $\underline{\mathrm{a}}(p, q)$ and $\underline{\mathrm{a}}(p+\mathrm{d} p, q+\mathrm{d} q)$ on the manifold surface. The matrix $\mathbb{G} \in \mathcal{R}^{2 \times 2}$ above is the metric tensor while its elements $g_{p p}, g_{q q}$, and $g_{p q}$ (note $g_{q p}=g_{p q}$ ) are known as the first fundamental coefficients and express the magnitudes and inner products of the parameter-curve tangent vectors. The following features may be readily established :

- I $\geq 0$ with equality when $\mathrm{d} p=\mathrm{d} q=0$.

- $g_{p p}, g_{q q} \geq 0$.

- By Schwarz's inequality : $\operatorname{det}(\mathbb{G}) \geq 0$.

- I is invariant under a parameter transformation.

- $g_{p p}, g_{p q}$ and $g_{q q}$ are variant under a parameter transformation.

The first fundamental form I is essential for the evaluation of lengths and areas on the manifold surface. For instance, let $\underline{\mathrm{a}}(t)=\underline{\mathrm{a}}(p(t), q(t))$ with $t_{1} \leq t \leq t_{2}$ be a regular curve on a surface $\underline{\mathrm{a}}=\underline{\mathrm{a}}(p, q)$. Then clearly the length of the curve is given by :

$$
\text { length of curve }=\int_{t_{1}}^{t_{2}}\left|\frac{\mathrm{d} a}{\mathrm{~d} t}\right| \mathrm{d} t=\int_{t_{1}}^{t_{2}} \sqrt{\frac{\mathrm{I}}{(\mathrm{d} t)^{2}}} \mathrm{~d} t
$$

It may be similarly shown that the area of a segment $W$ on the surface of a manifold $\underline{\mathrm{a}}=\underline{\mathrm{a}}(p, q)$ is given by :

$$
\text { area of segment }=\iint_{W} \sqrt{\operatorname{det}(\mathbb{G})} d p d q
$$

The significance of Equations (13) and (14) in relation to the intrinsic geometry of a surface will be clarified in the following sections.

\subsection{The Christoffel symbols}

In order to investigate the differential geometry of a surface $\underline{\mathrm{a}}(p, q)$, it is necessary to determine how the non-orthonormal basis $\mathbb{T}$ of the tangent plane varies from point to point on the array manifold. This information can be expressed in terms of the Christoffel symbols of first kind.

By using the matrix $\mathbb{T}=\left[\underline{\mathrm{a}}_{p}, \underline{\mathrm{a}}_{q}\right]$ and its two derivatives $\mathbb{T}_{p}=\partial \mathbb{T} / \partial p=\left[\underline{\mathrm{a}}_{p p}, \underline{\mathrm{a}}_{q p}\right]$ and $\mathbb{T}_{q}=\partial \mathbb{T} / \partial q=\left[\underline{\mathbf{a}}_{q q}, \underline{\mathrm{a}}_{q q}\right]$, the Christoffel symbols of the first kind represent the inner products between the tangent vectors $\underline{\mathrm{a}}_{p}, \underline{\mathrm{a}}_{q}$ and their derivatives, and are defined as

$$
\Gamma_{i, j k}=\operatorname{Re}\left\{\underline{\mathrm{a}}_{i}^{\mathrm{H}} \underline{\mathrm{a}}_{j k}\right\} \quad ; \quad i, j, k=p, q
$$


Thus there are eight Christoffel symbols of first kind forming the two Christoffel matrices of the first kind $\underline{\underline{\boldsymbol{\Gamma}}}_{1 p}$ and $\underline{\underline{\boldsymbol{\Gamma}}}_{1 q}$ defined as follows:

$$
\left\{\begin{array}{l}
\underline{\underline{\Gamma}}_{1 p}=\operatorname{Re}\left(\mathbb{T}^{\mathrm{H}} \mathbb{T}_{p}\right)=\left[\begin{array}{ll}
\Gamma_{p, p p} & \Gamma_{p, q p} \\
\Gamma_{q, p p} & \Gamma_{q, q p}
\end{array}\right] \\
\underline{\underline{\Gamma}}_{1 q}=\operatorname{Re}\left(\mathbb{T}^{\mathrm{H}} \mathbb{T}_{q}\right)=\left[\begin{array}{ll}
\Gamma_{p, p q} & \Gamma_{p, q q} \\
\Gamma_{q, p q} & \Gamma_{q, q q}
\end{array}\right]
\end{array}\right.
$$

where due to the symmetry inherent in differentiation, $\Gamma_{p, q p}=\Gamma_{p, p q}$ and $\Gamma_{q, q p}=\Gamma_{q, p q}$

Although the differential geometry of a surface can be completely described in terms of the above symbols, the process may be considerably simplified by the use of Christoffel symbols/matrices of the second kind which are related to the symbols of the first kind in the following way:

$$
\left\{\begin{array}{l}
\underline{\boldsymbol{\Gamma}}_{2 p}=\mathbb{G}^{-1} \underline{\boldsymbol{\Gamma}}_{1 p}=\left[\begin{array}{cc}
\Gamma_{p p}^{p} & \Gamma_{q p}^{p} \\
\Gamma_{p p}^{q} & \Gamma_{q p}^{q}
\end{array}\right] \\
\underline{\boldsymbol{\Gamma}}_{2 q}=\mathbb{G}^{-1} \underline{\boldsymbol{\Gamma}}_{1 q}=\left[\begin{array}{cc}
\Gamma_{p q}^{p} & \Gamma_{q q}^{p} \\
\Gamma_{p q}^{q} & \Gamma_{q q}^{q}
\end{array}\right]
\end{array}\right.
$$

Once again due to symmetry, $\Gamma_{p q}^{p}=\Gamma_{q p}^{p}$ and $\Gamma_{p q}^{q}=\Gamma_{q p}^{q}$. It is also customary to use superscripts to denote the elements of the inverse matrix $\mathbb{G}^{-1}$ :

$$
\mathbb{G}^{-1}=\left[\begin{array}{ll}
g^{p p} & g^{p q} \\
g^{q p} & g^{q q}
\end{array}\right]
$$

where clearly $g^{p p}=\frac{g_{q q}}{\operatorname{det}(\mathbb{G})}, g^{q q}=\frac{g_{p p}}{\operatorname{det}(\mathbb{G})}, g^{p q}=g^{q p}=\frac{-g_{p q}}{\operatorname{det}(\mathbb{G})}$.

It is obvious that all Christoffel symbols are functions only of the first fundamental coefficients $g_{i j}(i, j=p, q)$ and their derivatives, and for this reason they play a central role is expressing the intrinsic geometry of a surface, described next.

\subsection{Intrinsic geometry of a surface}

In order to define the intrinsic geometry of a surface, it is first necessary to appreciate the concept of an isometric mapping.

Definition $-A$ one-to-one mapping of a surface $\underline{\mathrm{a}}(p, q)$ on to a surface $\underline{\widehat{a}}(p, q)$ is called an isometric mapping or isometry if the length of an arbitrary curve $\underline{\mathrm{a}}(t)$ on $\underline{\mathrm{a}}$ is equal to the length of its image $\underline{\widehat{a}}(t)$ on $\underline{\widehat{a}}$.

Hence we may think of an isometry as the bending of a surface into a different shape without changing the distance (along the surface) between any of its points. Consequently, the "inhabitants" of such a surface would not be aware of any change at all, as their geometric measurements remain exactly the same. The combination of the 
above definition with Eq. (13) implies that a one-to-one mapping of a surface a on to another surface $\underline{\hat{a}}$ is an isometry iff at corresponding points the first fundamental coefficients remain unchanged, i.e. $g_{i j}=\widehat{g}_{i j} \forall i, j=p, q$. Thus there is no difference in the measurement of lengths, angles, and areas on isometric surfaces although the surfaces, when viewed from the embedding space, may have entirely different geometric shapes. An isometric mapping is by necessity both angle-preserving (conformal) and area-preserving (equiareal).

Definition - A property of a surface which remains invariant under an isometry is called an intrinsic property of the surface. The totality of the intrinsic properties of a surface is known as the intrinsic geometry of the surface.

Naturally a property of a surface is an intrinsic property if it only depends on the first fundamental coefficients (and hence the magnitudes and inner products of the tangents to the surface). The intrinsic geometry of a surface is completely independent of the space in which the surface is embedded and is built from the start solely on isometric invariants. Next, two important features of intrinsic geometry are briefly discussed, namely: Gaussian curvature and geodesic curvature.

\subsection{Gaussian curvature}

At every point on a surface $\underline{\mathrm{a}}(p, q)$, it is possible to define a real valued function $K_{\text {gauss }}$ called the Gaussian curvature, which - according to the Theorema Egregium of Gauss - is an intrinsic property of the surface and is representative of the local shape of the surface. The Gaussian curvature (also known as the curvature scalar) can be shown to be of the form [8]:

$$
K_{\text {gauss }}=\frac{1}{\operatorname{det}(\mathbb{G})}\left\{\frac{1}{2}\left(2 \frac{\partial^{2} g_{p q}}{\partial p \partial q}-\frac{\partial^{2} g_{p p}}{\partial q^{2}}-\frac{\partial^{2} g_{q q}}{\partial p^{2}}\right)+\sum_{i, j=p, q} g^{i j} \operatorname{det}\left(\mathbb{K}_{i j}\right)\right\}
$$

where

$$
\mathbb{K}_{i j}=\left[\begin{array}{ll}
\Gamma_{i, p q} & \Gamma_{i, p p} \\
\Gamma_{j, q q} & \Gamma_{j, q p}
\end{array}\right]
$$

The above expression may be more compactly represented in terms of the Christoffel symbols :

$K_{\text {gauss }}=\frac{1}{\operatorname{det}[\mathbb{G}]}\left\{\frac{\partial}{\partial p} \Gamma_{p, q q}-\frac{\partial}{\partial q} \Gamma_{p, q p}+\operatorname{col}_{2}\left\{\underline{\underline{\Gamma}}_{2 p}\right\}^{\mathrm{T}} \operatorname{col}_{1}\left\{\underline{\underline{\boldsymbol{\Gamma}}}_{1 q}\right\}-\operatorname{col}_{2}\left\{\underline{\underline{\boldsymbol{\Gamma}}}_{2 q}\right\}^{\mathrm{T}} \operatorname{col}_{1}\left\{\underline{\underline{\boldsymbol{\Gamma}}}_{1 p}\right\}\right\}$

where $\operatorname{col}_{i}\{$ matrix $\}$ denotes the $i^{\text {th }}$ column of the matrix. The sign of the Gaussian curvature at a point gives an indication of the local shape of the surface in that neighborhood in the following way:

- If $K_{\text {gauss }}>0$ Surface is locally elliptic.

- If $K_{\text {gauss }}<0$ Surface is locally hyperbolic.

- If $K_{\text {gauss }}=0$ Surface is locally either parabolic or planar (i.e. flat). 


\section{Example-1}

The following lists a number of surfaces in $\mathcal{R}^{3}$ and their respective Gaussian curvatures :
i) Sphere of radius $r$ :
$K_{\text {gauss }}=1 / \mathrm{r}^{2} \quad \Rightarrow$ Surface is elliptic at every point.
ii) Cylinder or Cone :
$K_{\text {gauss }}=0$
$\Rightarrow$ Surface is parabolic at every point.
iii) 2-D plane
$K_{\text {gauss }}=0$
$\Rightarrow$ Surface is flat at every point.

If the Gaussian curvature at a point on the manifold is a positive number, the local neighborhood of that point is equivalent to the local neighborhood of a point on a sphere of radius $\sqrt{1 / K_{\text {gauss }}}$. Also note that surfaces which have a zero Gaussian curvature at every point [e.g. Example-1 parts (ii) and (iii) above] are called developable surfaces.

Apart from defining the local shape of a surface, the Gaussian curvature has important implications with regards to isometric mappings. For example, two surfaces which are related by an isometric mapping must have the same Gaussian curvature at corresponding points (since $K_{\text {gauss }}$ is an intrinsic property). The converse of this is in general not true. However if two surfaces have the same constant Gaussian curvature, then any two sufficiently small neighborhoods of the surfaces are related by an isometric mapping. As a result, developable surfaces (for which $K_{\text {gauss }}=0$ ) are the only surfaces that can be mapped isometrically on to a plane. This feature will be used later in Section IV.

\subsection{Geodesic curves}

Geodesic curves on an arbitrary geometric surface generalize the notion of a straight line in Euclidean geometry. It is known that a straight line $\underline{\mathrm{a}}(t)=\underline{x}+t \underline{y}$ is characterized by zero curvature or acceleration $\kappa_{1}=\left|\underline{a}^{\prime \prime}\right|=0$. Following this line of reasoning, the intrinsic acceleration of a curve is defined as follows :

Definition - The geodesic curvature $\kappa_{g}$ (or intrinsic acceleration) of a curve $\underline{\mathrm{a}}(t)$ on a surface, is equal to the component of the curve's first curvature $\kappa_{1}$ along the tangent plane to the surface at every point along the curve.

The general expression for the geodesic curvature $\kappa_{g}$ of an arbitrary curve on a surface is rather involved but can be considerably simplified for the special case of constantparameter curves [9]:

$$
\begin{array}{ll}
p \text {-parameter curves }\left(\frac{\mathrm{d} q}{\mathrm{~d} s}=0\right) & \kappa_{g p}=\sqrt{\operatorname{det}[\mathbb{G}]} \frac{\Gamma_{p p}^{q}}{\left(g_{p p}\right)^{3 / 2}} \\
q \text {-parameter curves }\left(\frac{\mathrm{d} p}{\mathrm{~d} s}=0\right) & \kappa_{g q}=\sqrt{\operatorname{det}[\mathbb{G}]} \frac{-\Gamma_{q q}^{p}}{\left(g_{q q}\right)^{3 / 2}}
\end{array}
$$

Clearly the geodesic curvature is a function of the first fundamental coefficients only and is consequently preserved under an isometric mapping. This implies that an isometric mapping is by necessity also a geodesic mapping. 
Following the above definition, a geodesic curve may be defined as a curve whose geodesic curvature is zero at every point along its length. Since for a geodesic curve the direction of acceleration is always orthogonal to the surface, the inhabitants of a surface perceive no acceleration at all - for them the geodesic is a "straight" line.

It can also be shown that in the neighborhood of a point $A$ on a surface, there exists a unique geodesic through $A$ in any given direction. Consequently a unique geodesic joins point $A$ with every point $B$ in its neighborhood. Furthermore, the geodesic defines a unique arc of minimum length between neighboring points $A$ and $B$. The converse is also true in that if $\underline{a}(t)$ is an arc of minimum length between any two points on a surface, $\underline{a}(t)$ is a geodesic.

\section{Investigating the $\alpha$ - and $\beta$-parameter curves}

The differential geometry of the $\alpha$ - and $\beta$-curves are next investigated. Naturally, due to the symmetry of Eq. (7), the parameter curves are expected to exhibit similar characteristics.

\subsection{Arc lengths of the $\alpha$ - and $\beta$-parameter curves}

The most basic features of a curve $\underline{\mathrm{a}}(p)$ are its arc length $s$ and rate of change of arc length

$$
\dot{s}(p)=\mathrm{d} s / \mathrm{d} p=|\underline{\dot{a}}(p)|
$$

where $(\overline{)}$ denotes differentiation with respect to parameter $p$. Substituting Eq. (7) into Eq. (24) results in the following expressions for the rates of change of arc length of the $\alpha$ - and $\beta$-parameter curves (assuming $\phi \neq 0$ ) :

$$
\begin{cases}\dot{s}_{\alpha}(\alpha)=\pi|\underline{R}(\Theta)| \sin \alpha & \forall \alpha: \alpha_{\min }<\alpha<\alpha_{\max } \\ \dot{s}_{\beta}(\beta)=\pi\left|\underline{R}\left(\Theta+\frac{\pi}{2}\right)\right| \sin \beta & \forall \beta: \beta_{\min }<\beta<\beta_{\max }\end{cases}
$$

It is interesting to compare these with the corresponding expression for the $\theta$ parameter manifold of a linear array of isotropic sensors with locations $\underline{r}_{x}$ :

$$
\dot{s}_{\theta}(\theta)=\pi\left|\underline{r}_{x}\right| \sin \theta
$$

As will be seen later, the similarity is more than a coincidence.

Integrating the two parts of Eq.(25) over their respective limits results in the following expressions for the lengths of the two parameter curves :

$$
\left\{\begin{array}{l}
l_{\alpha \text {-curve }}=2 \pi|\underline{R}(\Theta)| \sin \beta \\
l_{\beta \text {-curve }}=2 \pi\left|\underline{R}\left(\Theta+\frac{\pi}{2}\right)\right| \sin \alpha
\end{array}\right.
$$

Clearly the $\alpha$ - (or $\beta$-) curve degenerates into a single point (i.e. a curve of zero length) whenever $\beta$ (or $\alpha$ ) approaches either 0 or $\pi$ radians. The shapes of the parameter curves are identified next through evaluation of their curvatures. 


\section{Theorem I : Curvatures of the $\alpha$ - and $\beta$-parameter curves}

The curvatures of the $\alpha$-and $\beta$-curves of the manifold of a planar array of isotropic sensors may written as a function of lower-order curvatures in the following way:

$$
\left\{\begin{array}{l}
\kappa_{\mathrm{i} \alpha}=\frac{1}{\kappa_{1 \alpha} \ldots \kappa_{(i-1) \alpha}}\left|\sum_{n=1}^{\operatorname{fix}\left(\frac{i}{2}\right)+1}(-1)^{n-1} h_{i, n} \underline{\widetilde{R}}(\Theta)^{i+3-2 n}\right| \\
\kappa_{\mathrm{i} \beta}=\frac{1}{\kappa_{1 \beta} \ldots \kappa_{(i-1) \beta}}\left|\sum_{n=1}^{\operatorname{fix}\left(\frac{i}{2}\right)+1}(-1)^{n-1} b_{i, n} \underline{\widetilde{R}}\left(\Theta+\frac{\pi}{2}\right)^{i+3-2 n}\right|
\end{array}\right.
$$

where $\kappa_{(i-1) \alpha}, \kappa_{(i-1) \beta} \neq 0$ and the polynomial coefficients are given by:

$$
\begin{cases}h_{i, n}=h_{i-1, n}+\kappa_{(i-1) \alpha}^{2} h_{i-2, n-1} ; & h_{i, 1}=1 \\ b_{i, n}=b_{i-1, n}+\kappa_{(i-1) \beta}^{2} b_{i-2, n-1} ; & b_{i, 1}=1\end{cases}
$$

with the initial conditions that:

$$
\kappa_{1 \alpha}=\left|\underline{\widetilde{R}}^{2}(\Theta)\right| \quad \kappa_{1 \beta}=\left|\underline{\widetilde{R}}^{2}\left(\Theta+\frac{\pi}{2}\right)\right|
$$

where, following the usual notation, $\underline{\widetilde{R}}=\frac{R}{|\underline{R}|}$.

The recursive Equations of (28) are similar in form, to those derived for the curvatures of the $\theta$-parameter manifold of a linear array of isotropic sensors [1], with the difference that $\underline{R}(\Theta)$ and $\underline{R}\left(\Theta+\frac{\pi}{2}\right)$ have taken on the role of the sensor location vector $\underline{r}$ of the linear array. Consequently, Theorem I can be proved in a similar fashion to this described in [1].

The curvatures and hence the shapes of the $\alpha, \beta$-curves depend strongly on $\Theta$, reminiscent of the drastic variations in array performance which can be observed along different lines of azimuth. Theorem-I is the centre-piece of this investigation and, as will be demonstrated next, has some far-reaching implications.

Corollary-1 : The $\alpha$ and $\beta$-curves are both hyperhelical.

This is apparent from Eq. (28) which indicate that curvatures $\kappa_{\mathrm{i} \alpha}$ and $\kappa_{\mathrm{i} \beta}$ are independent of $\alpha$ and $\beta$ respectively and are only functions of the array geometry projected along lines of azimuth at $\Theta$ and $\Theta+\frac{\pi}{2}$.

Corollary-2 : All members of the family of $\alpha$ - (or $\beta$-) curves are identical (congruent) apart from their length and position in complex $N$-space. 
This again follows from Eq. (28) which indicate that curvatures $\kappa_{\mathrm{i} \alpha}$ and $\kappa_{\mathrm{i} \beta}$ are independent of $\beta$ and $\alpha$ respectively (note the change in order). The above result means that the whole of the manifold surface may be fully covered by the simple translation of either of the two individual curves described by curvatures $\kappa_{\mathrm{i} \alpha}$ and $\kappa_{\mathrm{i} \beta}$.

Corollary-3 : The $\alpha$ - and $\beta$-curves are identical to the $\theta$-parameter manifold of a linear array of isotropic sensors with locations $\underline{R}(\Theta)$ and $\underline{R}\left(\Theta+\frac{\pi}{2}\right)$ respectively.

Fig. (3) illustrates the $\alpha-\beta$ parametrization of the array manifold in the special case of $\Theta=0$. Notice that for $\Theta=0$, cone angles are measured with respect to the $x$ and $y$ axes and consequently, according to Corollary -3 , the $\alpha$ - and $\beta$-curves are identical to the $\theta$-parameter manifolds of linear arrays with sensor locations $\underline{R}(0)=\underline{r}_{x}$ and $\underline{R}\left(\frac{\pi}{2}\right)=\underline{r}_{y}$ respectively.

Although the hyperhelical $\alpha / \beta$-coordinate system shown above covers the whole of the manifold surface and is sufficient to fully define the manifold shape, it is not unique. In fact, to understand the behaviour of the array for all possible directions of arrival it is necessary to consider the hyperhelical coordinates corresponding to all values of $\Theta$ from 0 to $\frac{\pi}{2}$.

The above concept may be more easily appreciated by observing Fig. (4) which shows the effect of a non-zero value of $\Theta$. It should be stressed that, despite the appearance of Fig. (4), the use of various values of $\Theta$ is not equivalent to a trivial rotation of the parameter curves, since their differential geometry is a function of $\Theta$ (Corollary-3).

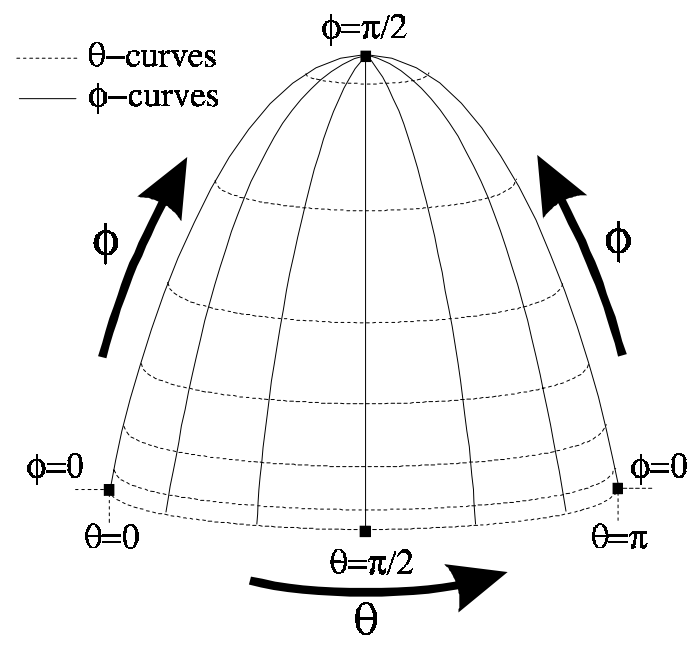

(a)

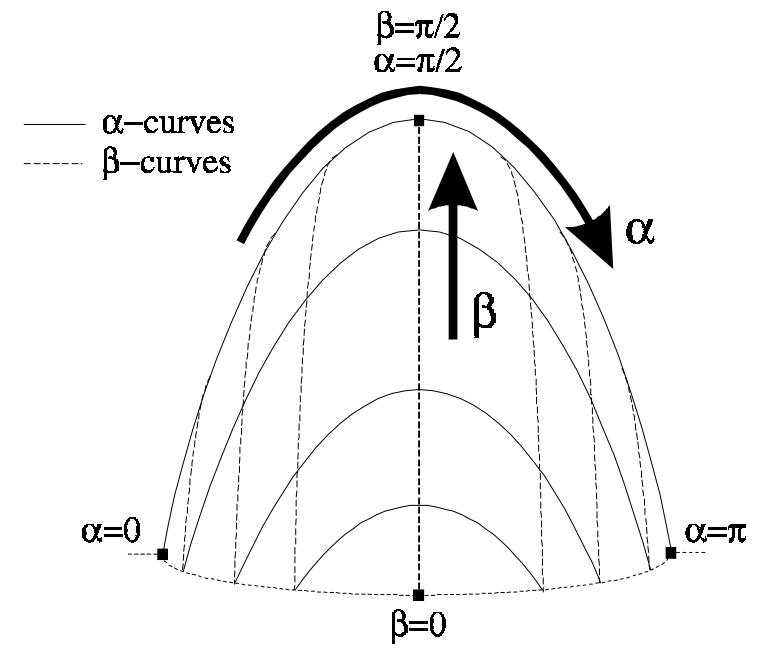

(b)

Fig.(3) : a) Polar coordinates resulting from azimuth-elevation parametrization of the manifold surface.

b) Grid coordinates resulting from cone-angle parametrization of the manifold surface with $\Theta=0$. 


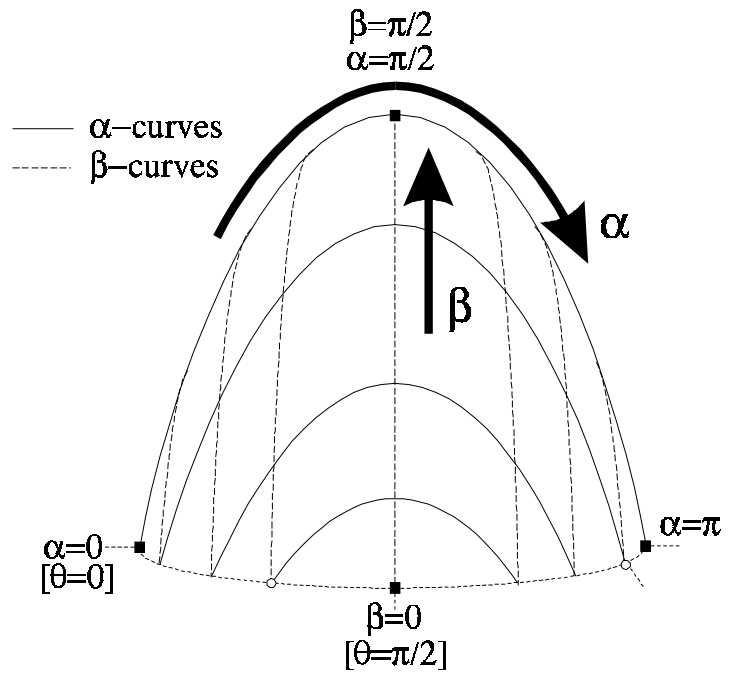

(a) $\Theta=0$

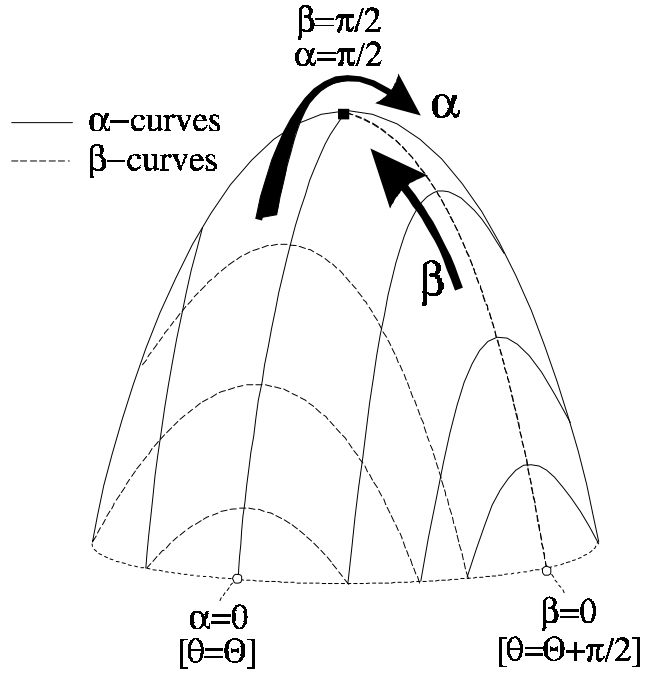

(b) $\Theta<\pi / 2$

Fig.(4) : $\quad$ a) Hyperhelical $\alpha / \beta$ coordinate system with $\Theta=0$.

b) Hyperhelical $\alpha / \beta$ coordinate system with $\Theta<\pi / 2$.

Figures (3) and (4) also reveal a clear relationship between the $\alpha / \beta$-curves and the hyperhelical $\phi$-curves, which will be stated as a final corollary :

Corollary-4: Each member of the $\alpha$-curve family is identical in shape to the combination of the $\phi$-curves corresponding to $\theta=\Theta$ and $\theta=\Theta+\pi$. Similarly each member of the $\beta$-curve family is identical to the combination of the $\phi$-curves at $\theta=\Theta+\frac{\pi}{2}$ and $\theta=\Theta+\frac{3 \pi}{2}$.

\section{Intrinsic Geometry of the Array Manifold}

As might be expected, the intrinsic shape of a manifold surface, as defined by its Gaussian curvature, is invariant under any parameter transformation. Here, however, it is demonstrated that the study of manifold geometry is considerably simplified as a result of $\alpha-\beta$ parametrization, once again confirming the advantages of cone angles over azimuth and elevation.

As usual, a study of intrinsic geometry begins with the specification of a metric tensor $\mathbb{G}$ and the evaluation of the first fundamental coefficients $g_{p q}$ and the Christoffel symbols. From Eq. (8) for the array manifold and the definitions of Section (2), it can be shown that:

$$
\begin{aligned}
& g_{\alpha \alpha}=\left|\underline{\mathrm{a}}_{\alpha}\right|^{2}=(\pi \underline{\mid R}(\Theta) \mid \sin \alpha)^{2} \\
& g_{\beta \beta}=\left|\underline{\mathrm{a}}_{\beta}\right|^{2}=\left(\pi\left|\underline{R}\left(\Theta+\frac{\pi}{2}\right)\right| \sin \beta\right)^{2} \\
& \mathrm{~g}_{\alpha \beta}=g_{\beta \alpha}=\operatorname{Re}\left(\underline{\mathrm{a}}_{\alpha}^{\mathrm{H}} \underline{\mathrm{a}}_{\beta}\right)=\pi^{2} \underline{R}(\Theta)^{\mathrm{T}} \underline{R}\left(\Theta+\frac{\pi}{2}\right) \sin \alpha \sin \beta
\end{aligned}
$$


where, as usual, $\underline{\mathrm{a}}_{\alpha}=\frac{\partial \mathrm{a}}{\partial \alpha}$ and $\underline{\mathrm{a}}_{\beta}=\frac{\partial \mathrm{a}}{\partial \beta}$.

Whenever $\alpha$ or $\beta$ equal 0 or $\pi$, the matrix $\mathbb{G}$ of the first fundamental coefficients becomes singular. At such singularities, one or both of the tangent vectors $\underline{\mathrm{a}}_{\alpha}$ or $\underline{\mathrm{a}}_{\beta}$ vanish and so the tangent plane is no longer defined. Fortunately the requirement $\phi \neq 0$ (for the independence of $\alpha$ and $\beta$ ) established at the outset avoids these singularities. Also note that the grid parametrization avoids the singularity at the apex $\left(\phi=\frac{\pi}{2}\right)$ which exists with $\theta-\phi$ parametrization and hence is more suitable for the study of the manifold in that region.

It is a simple matter to confirm that for balanced-symmetric arrays $\underline{R}(\Theta)^{\mathrm{T}} \underline{R}\left(\Theta+\frac{\pi}{2}\right)=0 \forall \Theta$ and so the tangent vectors $\underline{\mathrm{a}}_{\alpha}$ and $\underline{\mathrm{a}}_{\beta}$ are strictly orthogonal. For such arrays matrix $\mathbb{G}$ is diagonal and the hyperhelical $\alpha-\beta$ curves form an orthogonal coordinate system.

The other variables required for the study of intrinsic geometry are the Christoffel Symbols. From the definitions of Section (3) it can be shown that if $\mathbb{T}=\left[\underline{a}_{\alpha}\right.$ $\left.\underline{\mathrm{a}}_{\beta}\right] \in \mathcal{C}^{N \times 2}$ then

$$
\left\{\begin{array}{l}
\underline{\Gamma}_{1 \alpha}=\operatorname{Re}\left(\mathbb{T}^{\mathrm{H}} \mathbb{T}_{\alpha}\right)=\left[\begin{array}{ll}
\Gamma_{\alpha, \alpha \alpha} & \Gamma_{\alpha, \beta \alpha} \\
\Gamma_{\beta, \alpha \alpha} & \Gamma_{\beta, \beta \alpha}
\end{array}\right]=\cot \alpha\left[\begin{array}{ll}
\mathrm{g}_{\alpha \alpha} & 0 \\
\mathrm{~g}_{\beta \alpha} & 0
\end{array}\right] \\
\underline{\Gamma}_{1 \beta}=\operatorname{Re}\left(\mathbb{T}^{\mathrm{H}} \mathbb{T}_{\beta}\right)=\left[\begin{array}{ll}
\Gamma_{\alpha, \alpha \beta} & \Gamma_{\alpha, \beta \beta} \\
\Gamma_{\beta, \alpha \beta} & \Gamma_{\beta, \beta \beta}
\end{array}\right]=\cot \beta\left[\begin{array}{ll}
0 & \mathrm{~g}_{\alpha \beta} \\
0 & \mathrm{~g}_{\beta \beta}
\end{array}\right]
\end{array}\right.
$$

and :

$$
\left\{\begin{array}{l}
\underline{\underline{\Gamma}}_{2 \alpha}=\mathbb{G}^{-1} \operatorname{Re}\left(\mathbb{T}^{\mathrm{H}} \mathbb{T}_{\alpha}\right)=\left[\begin{array}{cc}
\Gamma_{\alpha \alpha}^{\alpha} & \Gamma_{\beta \alpha}^{\alpha} \\
\Gamma_{\alpha \alpha}^{\beta} & \Gamma_{\beta \alpha}^{\beta}
\end{array}\right]=\cot \alpha\left[\begin{array}{ll}
1 & 0 \\
0 & 0
\end{array}\right] \\
\underline{\underline{\Gamma}}_{2 \beta}=\mathbb{G}^{-1} \operatorname{Re}\left(\mathbb{T}^{\mathrm{H}} \mathbb{T}_{\beta}\right)=\left[\begin{array}{cc}
\Gamma_{\alpha \beta}^{\alpha} & \Gamma_{\beta \beta}^{\alpha} \\
\Gamma_{\alpha \beta}^{\beta} & \Gamma_{\beta \beta}^{\beta}
\end{array}\right]=\cot \beta\left[\begin{array}{ll}
0 & 0 \\
0 & 1
\end{array}\right]
\end{array}\right.
$$

Note that the Christoffel matrices are quite sparse (even more so in the case of balanced-symmetric arrays where $\mathrm{g}_{\alpha \beta}=\mathrm{g}_{\beta \alpha}=0$ ), which is a desirable feature and simplifies the differential geometry considerably.

\section{Theorem II : Geodesic Curvatures of the $\alpha$ - and $\beta$-Curves}

The hyperhelical $\alpha$ - and $\beta$-curves form a geodesic coordinate system for all values of $\Theta$.

\section{Proof :}

Following the definitions of Section II, the geodesic curvatures of the $\alpha$ - and $\beta$-curves are given by : 


$$
\left\{\begin{array}{l}
\kappa_{\mathrm{g} \alpha}=\sqrt{\operatorname{det}[\mathbb{G}]} \frac{\Gamma_{\alpha \alpha}^{\beta}}{\left(\mathrm{g}_{\alpha \alpha}\right)^{3 / 2}} \\
\kappa_{\mathrm{g} \beta}=-\sqrt{\operatorname{det}[\mathbb{G}]} \frac{\Gamma_{\beta \beta}^{\alpha}}{\left(\mathrm{g}_{\beta \beta}\right)^{3 / 2}}
\end{array}\right.
$$

Eq. (35) clearly indicates that $\Gamma_{\alpha \alpha}^{\beta}=\Gamma_{\beta \beta}^{\alpha}=0$, therefore $\kappa_{\mathrm{g} \alpha}=\kappa_{\mathrm{g} \beta}=0 \quad \forall \alpha, \beta$; i.e. the parameter curves are both geodesic.

The geodesic nature of the cone angle parameter curves and their length-minimizing characteristics will be exploited later when examining isometric mappings of the manifold and array performance.

\section{Theorem III : Gaussian Curvature Of The Array Manifold}

The Gaussian curvature $K_{\text {gauss }}$ of the two-parameter manifold of a planar array of $N$ isotropic sensors is constant and equal to zero at every point.

\section{Proof :}

Following the definition of Section II and the Christoffel symbol matrices defined in Section (3), the Gaussian curvature of the array manifold may be written as :

$$
\begin{aligned}
K_{\text {gauss }} & =\frac{1}{\operatorname{det}[\mathbb{G}]}\left\{\frac{\partial}{\partial \alpha} \Gamma_{\alpha, \beta \beta}-\frac{\partial}{\partial \beta} \Gamma_{\alpha, \beta \alpha}+\operatorname{col}_{2}\left\{\underline{\underline{\Gamma}}_{2 \alpha}\right\}^{\mathrm{T}} \operatorname{col}_{1}\left\{\underline{\underline{\Gamma}}_{1 \beta}\right\}-\operatorname{col}_{2}\left\{\underline{\underline{\Gamma}}_{2 \beta}\right\}^{\mathrm{T}} \operatorname{col}_{1}\left\{\underline{\underline{\Gamma}}_{1 \alpha}\right\}\right\} \\
& =\frac{1}{\operatorname{det}[\mathbb{G}]}\left\{\cot \beta \frac{\partial}{\partial \alpha} g_{\alpha \beta}-0+0-\cot \beta \cot \alpha g_{\beta \alpha}\right\} \\
& =\frac{1}{\operatorname{det}[\mathbb{G}]}\left\{\cot \beta \cot \alpha g_{\alpha \beta}-\cot \beta \cot \alpha g_{\beta \alpha}\right\}=0
\end{aligned}
$$

The theorem implies that the manifold is locally parabolic in $\mathcal{C}^{N}$, in the same way that a circular cylinder or cone is locally parabolic in Euclidean space $\mathcal{R}^{3}$.

The most significant implication of Theorem III is that the array manifold, by virtue of its zero Gaussian curvature, is a developable surface and hence can be isometrically mapped on to any other surface of zero curvature, say a plane in $\mathcal{R}^{2}$. The result of such a mapping would be a consistent two-dimensional representation (development) of the manifold in real space. A development may be readily evaluated by noting that if $\underline{\mathrm{a}}(p), \underline{\mathrm{b}}(p) \in \mathcal{C}^{N}$ are arbitrary curves on the manifold surface and $\underline{\mathrm{a}}_{\mathrm{d}}(p), \underline{\mathrm{b}}_{\mathrm{d}}(p) \in \mathcal{R}^{2}$ are their respective images on the development, then since an isometric mapping is by necessity both geodesic and conformal :

- Length of $\underline{\mathrm{a}}_{\mathrm{d}}(p) \in \mathcal{R}^{2} \quad=$ Length of $\underline{\mathrm{a}}(p) \in \mathcal{C}^{N}$

- Curvature of $\underline{\mathrm{a}}_{\mathrm{d}}(p) \in \mathcal{R}^{2} \quad=$ Geodesic curvature of $\underline{\mathrm{a}}(p) \in \mathcal{C}^{N}$

- $\angle \underline{\mathrm{a}}_{\mathrm{d}}(p), \underline{\mathrm{b}}_{\mathrm{d}}(q) \in \mathcal{R}^{2} \quad=\angle \underline{\mathrm{a}}(p), \underline{\mathrm{b}}(q) \in \mathcal{C}^{N}$ 
The above features may be demonstrated by the following example.

\section{Example-2}

Consider the developments of the manifolds of two array configurations when parametrized with cone angles $\alpha$ and $\beta$ with $\Theta=0$ (recall that $\underline{R}(0)=\underline{r}_{x}$ and $\left.\underline{R}(\pi / 2)=\underline{r}_{y}\right)$. The array structures are indicated in Figures (5a) and (5b) with the respective developments in Figures (5c) and (5d). Cone angles $\alpha$ and $\beta$ are considered in steps of $10^{\circ}$ from $0^{\circ}$ to $180^{\circ}$.

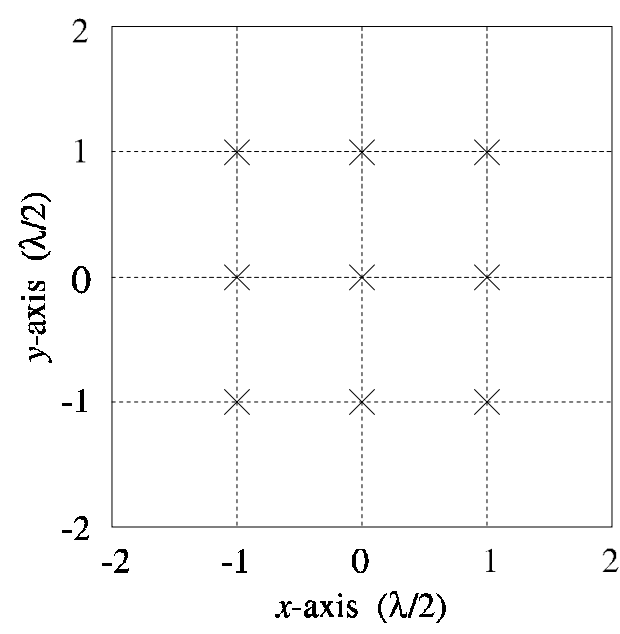

(a)

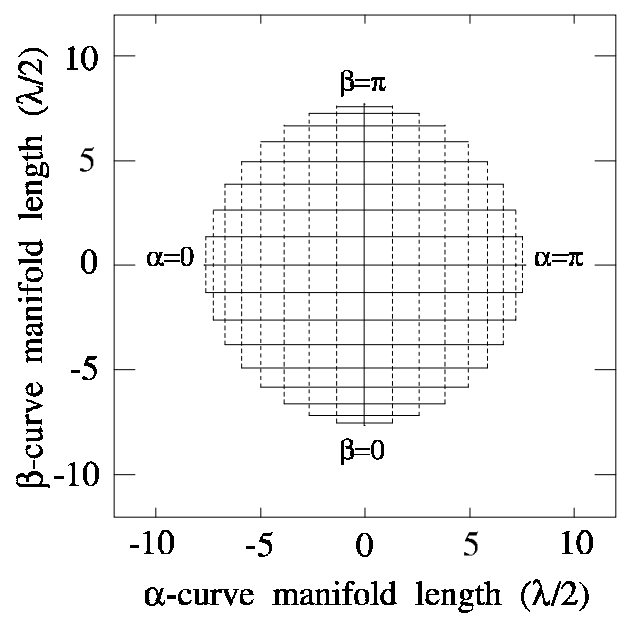

(c)

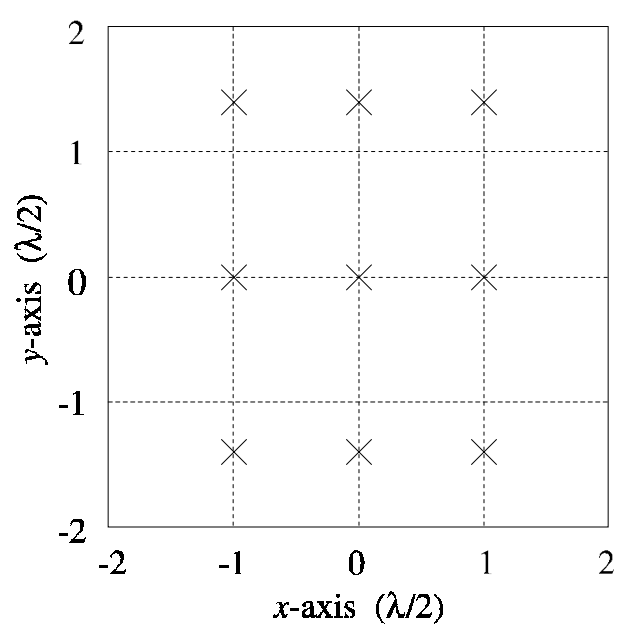

(b)

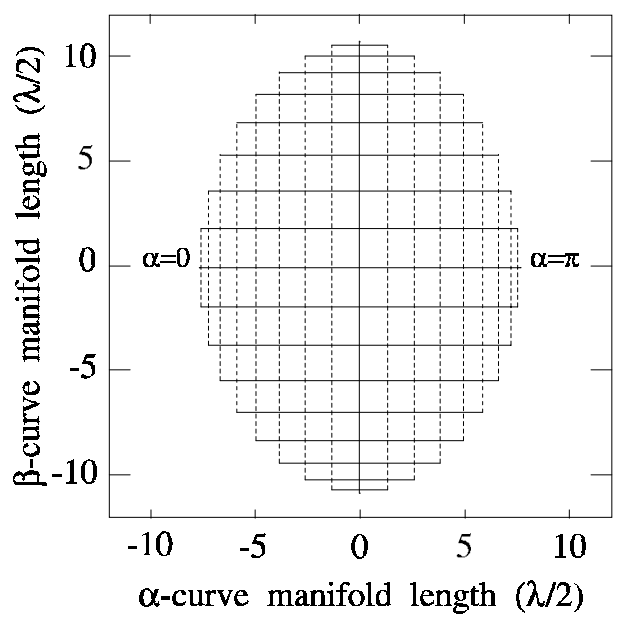

(d)

Fig.(5) : (a) Square-grid array structure, $\underline{\mathrm{r}}_{\mathrm{y}}=\underline{\mathrm{r}}_{\mathrm{x}}$ (balanced-symmetric).

(b) Rectangular-grid array structure $\underline{r}_{\mathrm{y}}=1.4 \underline{\mathrm{r}}_{\mathrm{x}}$ (unbalanced-symmetric).

(c) (d) Developments of the manifolds of arrays in Figures (a) and (b) respectively when the manifolds are parametrized by cone angles $\alpha$ and $\beta$ where $\Theta=0$.

Since both arrays are symmetric (i.e. $\underline{r}_{x}^{\mathrm{T}} \underline{r}_{y}=0$ ), the $\alpha$ - and $\beta$-curves are orthogonal and geodesic, as a result of which their images on the development are orthogonal 
straight lines (geodesics) and of length $2 \pi\left|\underline{r}_{x}\right| \sin \beta$ and $2 \pi\left|\underline{r}_{y}\right| \sin \alpha$ respectively. Note that the second array geometry is unbalanced $\left(\left|\underline{r}_{y}\right|>\left|\underline{r}_{x}\right|\right)$ and consequently the envelope of the development is elliptical rather than circular.

\section{Applications Of Cone-Angle Parametrization}

Having investigated the nature of the $\alpha / \beta$ parameter curves, it is now possible to examine their implications in connection with the direction-finding capabilities of a planar array of sensors.

\subsection{Further insight into the direction finding performance of a planar array}

In reference [6,7] the accuracy, detection and resolution capabilities of an array of sensors and their dependence on the differential geometry of manifold parameter curves were investigated in some detail. It was seen that for sufficiently close emitters, the array performance is a function of the local shape of a manifold curve as specified by its length and first curvature.

It was consequently deduced that while performance varies with azimuth $\theta$ in accordance with the array configuration, its variation with elevation $\phi$ obeys simple sinusoidal laws irrespective of the array. While these deductions are completely valid, examination of array performance in terms of cone angles can provide an alternative, and perhaps a clearer picture of the nature of a planar array.

\section{Theorem IV}

The capability of a planar array in estimating, detecting and resolving cone angles $\alpha_{1}$ and $\alpha_{2}$ (measured relative to line of azimuth $\Theta$ ) of two emitters at bearings $\left(\alpha_{1}, \beta\right)$ and $\left(\alpha_{2}, \beta\right)$ is

i) independent of the value of $\beta$.

ii) identical to its capability in estimating, detecting and resolving the elevations of two emitters at (azimuth,elevation) bearings :

Proof :

$$
\left\{\begin{array}{l}
\left(\theta=\Theta, \phi=\alpha_{1}\right) \text { and }\left(\theta=\Theta, \phi=\alpha_{2}\right) \text { if } \alpha_{1}, \alpha_{2}<\frac{\pi}{2} \\
\text { or } \\
\left(\theta=\Theta, \phi=\pi-\alpha_{1}\right) \operatorname{and}\left(\theta=\Theta, \phi=\pi-\alpha_{2}\right) \text { if } \alpha_{1}, \alpha_{2}>\frac{\pi}{2}
\end{array}\right.
$$

i) According to Corollary- 1 of Theorem I, all members of the family of $\alpha$-curves are identical, i.e. their differential geometry is independent of $\beta$.

ii) From Corollary- 4 of Theorem-I, each member of the $\alpha$-curve family is identical to the combination of the $\phi$-curves corresponding to $\theta=\Theta$ and $\theta=\Theta+\pi$. This implies that $\alpha$-estimation and $\phi$-estimation performance along line of azimuth $\Theta$ must by necessity be equivalent. Furthermore, since the $\phi$-curves at $\theta=\Theta$ and $\theta=\Theta+\pi$ are mirror images, we need only consider the former.

Naturally, the roles of $\alpha$ and $\beta$ may be interchanged in Theorem IV by simply replacing $\Theta$ with $\Theta+\frac{\pi}{2}$.

Part $(i)$ of the above theorem suggests an underlying "conic" behaviour in the performance of a planar array which could not be observed by consideration of 
azimuth and elevation alone. Fig. (6a-b) illustrate this behaviour by showing the loci of two directions of arrival separated by $\Delta \alpha=2^{\circ}\left(\alpha_{1}=68^{\circ}, \alpha_{2}=70^{\circ}\right)$ for all possible values of $\beta$ and the corresponding $\theta-\phi$ values.

Part $(i i)$ of Theorem IV indicates that the theory of $\phi$-estimation array performance is directly applicable to all corresponding cone angles (once sensor directivity is accounted for). In particular, one might immediately deduce that the $\alpha$ - and $\beta$ estimation performances of a planar array of isotropic sensors are at their peaks when $\alpha=\frac{\pi}{2}$ and $\beta=\frac{\pi}{2}$ respectively, and degrade sinusoidally as $\alpha$ and $\beta$ approach either 0 or $\pi$.

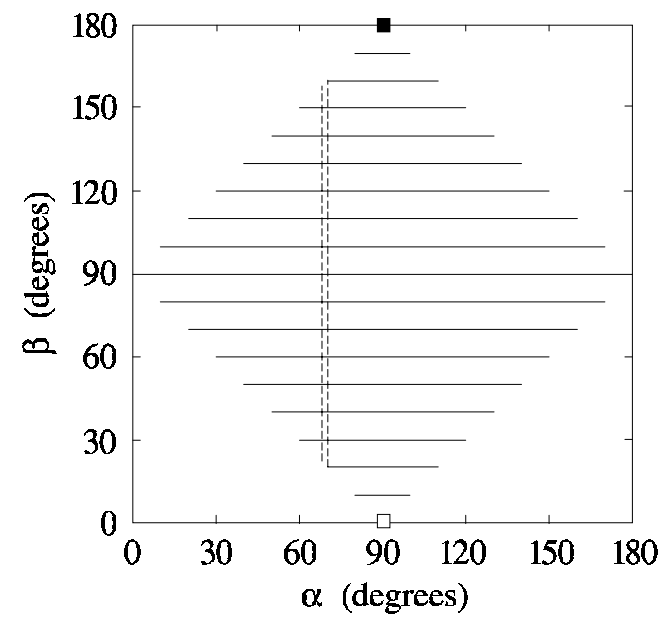

(a)

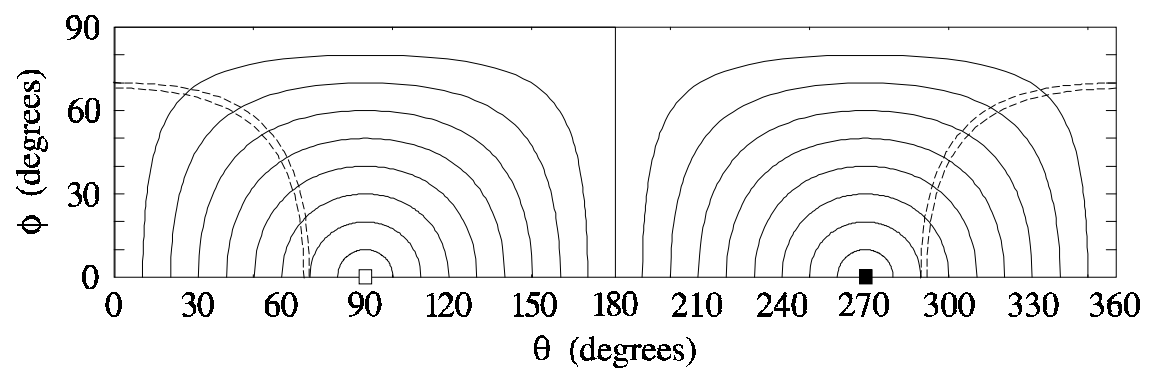

(b)

- - - - Direction of Arrival Loci

Fig.(6) : a) The loci of two directions of arrival separated by $\Delta \alpha=2^{\circ}$ for all values of $\beta$. According to Theorem IV part-i, the direction finding (estimation, detection and resolution) capability of a planar array is the same in the case of any of the above direction-of-arrival pairs (i.e. is independent of $\beta$ ).

b) The corresponding direction-of-arrival loci of part (a) on the $\theta-\phi$ parameter plane reveals the conic nature of the array performance. 


\subsection{Planar Array Performance For Emitters At Different Azimuths And Elevations} In general the array performance is investigated under the scenario of two emitters closely spaced either in azimuth (equal elevations) or in elevation (equal azimuths). Here it is demonstrated how, based on cone parametrization, the analysis techniques used can be adapted to cope with more realistic cases where the emitters are located at $\left(\theta_{1}, \phi_{1}\right)$ and $\left(\theta_{1}+\Delta \theta, \phi_{1}+\Delta \phi\right)$; i.e. neither of the two bearing parameters are common.

The situation is depicted in Fig. (7a). It is intuitively apparent that the problem essentially involves the identification of the unique $^{1}$ geodesic curve (curve of minimum length) which joins points $P$ and $Q$ on the manifold surface. The arc length and curvatures of such a curve would then dictate the ultimate capabilities of the array in estimating the directions of arrival.

In what follows, it is demonstrated that it is always possible, via a proper choice of $\Theta$, to identify a hyperhelical/geodesic curve which connects any two arbitrary points on the manifold surface. Consider two emitters with arbitrary wavenumber vectors $\underline{k}_{1}$ and $\underline{k}_{2}$ as shown in Fig. (7b).

Clearly any two such vectors would have to lie on a cone of constant (say) $\alpha$ about a line of azimuth $\Theta$. Therefore the geodesic curve which joins manifold vectors $\underline{\mathrm{a}}\left(\theta_{1}, \phi_{1}\right)$ and $\underline{\mathrm{a}}\left(\theta_{2}, \phi_{2}\right)$ must also be a hyperhelical $\beta$-curve.

a)

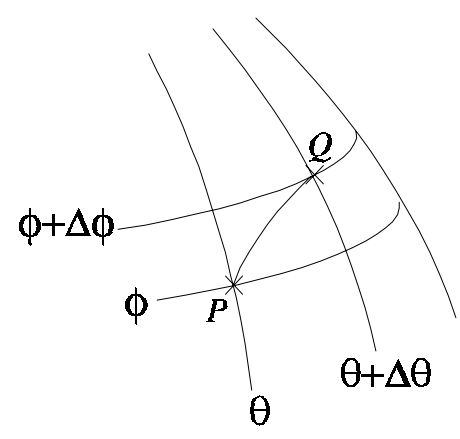

b)

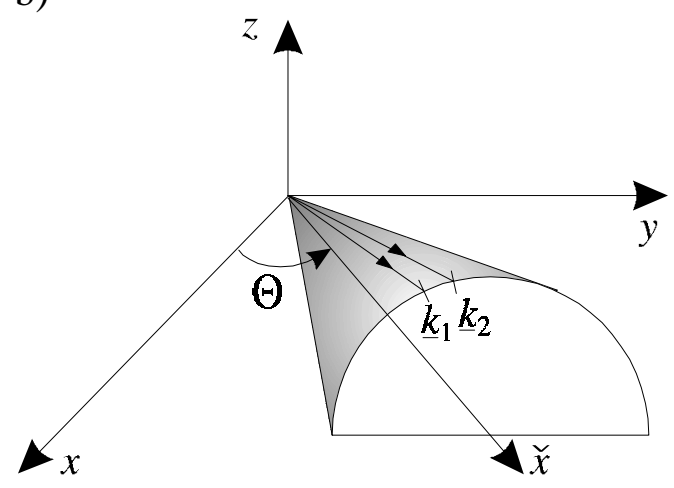

Fig.(7) : a) Array manifold points for two emitters at different Azimuths and Elevations.

b) wavenumber vectors $k_{1}$ and $k_{2}$ for two arbitrary emitters.

The value of $\Theta$ of course is decided by the directions of arrival. Denoting the rotated $\mathrm{x}$-axis by unit vector $\underline{v}=[\cos \Theta, \sin \Theta, 0]^{\mathrm{T}}$, for equal $\alpha$ cone angles :

\footnotetext{
${ }^{1}$ On the neighbourhood of a point $A$ on the surface of class $\geq 3$ (differentiable more than three times), there exists one and only one geodesic through $\mathrm{A}$ in any given direction.
} 
$\underline{k}_{1}^{\mathrm{T}} \underline{\underline{v}}=\underline{k}_{2}^{\mathrm{T}} \underline{\underline{v}} \Rightarrow \quad \Theta=\tan ^{-1}\left\{\frac{\cos \phi_{1} \cos \theta_{1}-\cos \phi_{2} \cos \theta_{2}}{\cos \phi_{2} \sin \theta_{2}-\cos \phi_{1} \sin \theta_{1}}\right\}$

The cone angles are then given by:

$$
\left\{\begin{array}{l}
\alpha=\cos ^{-1}\left(\frac{1}{\pi} \underline{k}_{1}^{\mathrm{T}} \underline{\underline{x}}\right) \\
\beta_{i}=\cos ^{-1}\left(\frac{1}{\pi} \underline{k}_{i}^{\mathrm{T}} \underline{y}\right) \quad i=1,2
\end{array}\right.
$$

where $\underline{v}=[-\sin \Theta, \cos \Theta, 0]^{\mathrm{T}}$.

\section{Example-3}

Consider a 24-element Y-shaped array with sensor locations of $[8,22,38,57,79,105,136,170] / 15$ half-wavelengths along each branch (branches separated by $120^{\circ}$ ). Determine the ultimate SNR required to resolve two unit-powered emitters located at azimuths $\theta_{1}=35^{\circ}$ and $\theta_{2}=36^{\circ}$ and elevations $\phi_{1}=58^{\circ}$ and $\phi_{2}=56.5^{\circ}$ over an observation interval of 100 snapshots $(L=100)$.

According to Equations (38) and (39) :

$$
\begin{array}{ll}
\Theta=-31.290^{\circ} & \beta_{1}=60.975^{\circ} \\
\alpha=77.697^{\circ} & \beta_{2}=59.393^{\circ}
\end{array}
$$

In other words, the geodesic curve joining the two manifold vectors is a hyperhelical $\beta$-curve $\left(12.303^{\circ} \leq \beta \leq 167.70^{\circ}\right)$ with the following features :

$$
\begin{gathered}
\dot{s}_{\beta}(\beta)=\pi\left|\underline{R}\left(58.710^{\circ}\right)\right| \sin \beta=67.819 \sin \beta \\
\kappa_{1 \beta}=\left|\underline{\widetilde{R}}^{2}\left(58.710^{\circ}\right)\right|=0.3718 \\
\sin \zeta_{\beta}=\sqrt{1-\frac{1}{\kappa_{1 \beta}^{2}}\left[\operatorname{sum}\left(\underline{\widetilde{R}}^{3}\left(58.710^{\circ}\right)\right)\right]^{2}}=0.8331
\end{gathered}
$$

in which case $\Delta s=\dot{s}_{\beta}\left(\frac{\beta_{1}+\beta_{2}}{2}\right) \Delta \beta=1.6247$. From Equ (39) in Reference [3]

$$
\mathrm{SNR}_{\beta \text {-RES }}=\frac{32}{L(\Delta s)^{4}\left(\widehat{\kappa}_{1 \beta}^{2}-\frac{1}{N}\right)}=0.85(-0.71 \mathrm{~dB})
$$

\subsection{Ambiguities in Planar Arrays}

The presence of ambiguities in bearing estimation appears as extraneous nulls (corresponding to non-existent emitters) in the DF spectrum of any direction-finding technique.

Recently two new techniques have been developed for calculating the ambiguous sets of directions related to linear array structures. The first technique has been reported in [4] and is based on the uniform partition of the manifold of a linear array by exploiting its differential geometry parameters. The second technique [5] is based on the partition of the array manifold using the roots of the function 


$$
\operatorname{tr}\left(\mathbb{C}^{x} \operatorname{expm}(s \mathbb{C})\right)
$$

where $x$ is an arbitary positive number and $\mathbb{C}$ is the Cartan Matrix, having the following structure :

$$
\mathbb{C} \triangleq\left[\begin{array}{ccccc}
0 & -\kappa_{1} & 0 & \cdots & 0 \\
\kappa_{1} & 0 & -\kappa_{2} & \cdots & 0 \\
0 & \kappa_{2} & 0 & \cdots & 0 \\
\vdots & \vdots & \vdots & \vdots & \vdots \\
0 & 0 & 0 & \kappa_{d-1} & 0
\end{array}\right]
$$

with $\kappa_{i}$ denoting the $i^{\text {th }}$ curvature. As a result of the Theorem I and its corollaries these techniques can be directly applied to planar arrays to discover and estimate new sets of ambiguous directions based on the partition of $\alpha$ and $\beta$ parameters curves.

\section{Conclusions}

In this study, cone angles $\alpha$ and $\beta$ were introduced as an alternative and more helpful method for the parametrization of the planar array manifold. It was shown that this results in two families of $\alpha$ - and $\beta$-parameter curves which are not only hyperhelical but are also geodesic and are closely related to the single-parameter manifolds of linear arrays. Furthermore, it was shown that the members within each family of parameter curves are identical in shape but are of different lengths.

Finally the performance analysis of a planar array structures and the array ambiguities were taken as two examples for demonstrating the significance of the above findings on the general Direction Finding problem. 


\section{References}

[1] Dacos I. and Manikas A., "The Use of Differential Geometry in Estimating the Manifold Parameters of a one - Dimensional Array of Sensors", Journal of The Franklin Institute, Engineering and Applied Mathematics , Vol.332B, No.3, pp307-332, 1995.

[2] Dowlut N. and Manikas A., "Array Design for Superresolution DirectionFinding Algorithms", International Symposium on Digital Signal Processing ISDSP-96, London, pp 38-43, June 1996.

[3] Manikas A., Karimi H.R. and Dacos I. , "Study of the Detection and Resolution Capabilities of One-Dimensional Array of Sensors by using Differential Geometry", IEE Proceedings on Radar, Sonar \& Navigation, vol. 141, No. 2, pp 83-92, April 1994.

[4] Proukakis C. and Manikas A., "Study of Ambiguities of Linear Arrays", IEEE Proceedings of ICASSP, vol IV, pp. 549-552, 1994.

[5] Manikas A. and Proukakis C., "Modeling, Identification and Estimation of Ambiguities in DF Systems", Imperial College EEED Internal Research Report AM-96-2, 1996.

[6] Karimi H.R and Manikas A., "The Manifold of Planar Arrays and its Effects on the Accuracy of Direction-Finding Systems", IEE Proceedings on Radar, Sonar \& Navigation, Vol.143, No.6, pp. 349-357, December 1996.

[7] Manikas A, Alexiou A. and Karimi H. R., "Comparison of the Ultimate Direction-Finding Capabilities of a Number of Planar Array Geometries", accepted for publication, IEE Proceedings on Radar, Sonar \& Navigation.

[8] Dacos I. and Manikas A., "Investigating the Manifold Parameters of a Non Linear Array of Omnidirectional Sensors. Part I : Arrays with Arbitrary 3Dimensional Geometry", Imperial College EEED Internal Research Report AM91-4, Part I, 28 pages, September 1991.

[9] Lipschutz M. M. , "Differential Geometry", McGraw-Hill, 1969. 